\title{
(I) \\ Incorporating ethical principles into clinical research protocols: a tool for protocol writers and ethics committees
}

\author{
Rebecca H Li, ${ }^{1}$ Mary C Wacholtz, ${ }^{2}$ Mark Barnes, ${ }^{1,3}$ Liam Boggs, ${ }^{4}$ Susan Callery- \\ D'Amico, ${ }^{5}$ Amy Davis, ${ }^{6}$ Alla Digilova, ${ }^{10}$ David Forster, ${ }^{7}$ Kate Heffernan, ${ }^{8}$ \\ Maeve Luthin, ${ }^{8}$ Holly Fernandez Lynch, ${ }^{9}$ Lindsay McNair, ${ }^{6}$ Jennifer E Miller, ${ }^{11}$ \\ Jacquelyn Murphy, ${ }^{12}$ Luann Van Campen, ${ }^{13}$ Mark Wilenzick, ${ }^{14}$ Delia Wolf, ${ }_{1}^{15}$ \\ Cris Woolston, ${ }^{16}$ Carmen Aldinger, ${ }_{1}^{1}$ Barbara E Bierer ${ }^{17}$
}

\begin{abstract}
- Additional material is published online only. To view, please visit the journal online (http://dx.doi.org/10.1136/ medethics-2014-102540).

For numbered affiliations see end of article.
\end{abstract}

\section{Correspondence to} Dr Rebecca Li, Multi-Regional Clinical Trials Center of Brigham and Women's Hospital and Harvard, 14 Story Street, 4th Floor, Cambridge, MA 02138, USA

RLi@bwh.harvard.edu

Received 27 October 2014 Revised 14 September 2015 Accepted 14 December 2015 Published Online First 25 January 2016
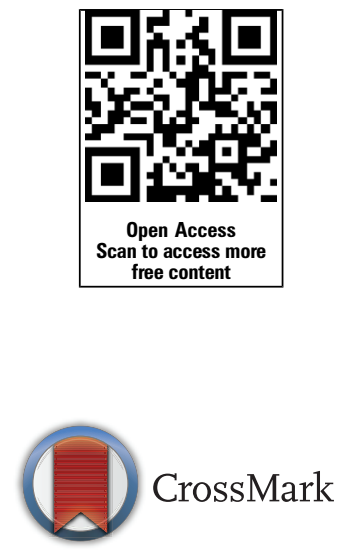

To cite: $\mathrm{Li} R \mathrm{RH}$

Wacholtz MC, Barnes M,

et al. J Med Ethics

2016:42:229-234.

\section{ABSTRACT}

A novel Protocol Ethics Tool Kit ('Ethics Tool Kit') has been developed by a multi-stakeholder group of the Multi-Regional Clinical Trials Center of Brigham and Women's Hospital and Harvard. The purpose of the Ethics Tool Kit is to facilitate effective recognition, consideration and deliberation of critical ethical issues in clinical trial protocols. The Ethics Tool Kit may be used by investigators and sponsors to develop a dedicated Ethics Section within a protocol to improve the consistency and transparency between clinical trial protocols and research ethics committee reviews. It may also streamline ethics review and may facilitate and expedite the review process by anticipating the concerns of ethics committee reviewers. Specific attention was given to issues arising in multinational settings. With the use of this Tool Kit, researchers have the opportunity to address critical research ethics issues proactively, potentially speeding the time and easing the process to final protocol approval.

The principal goal of clinical research, even when benefiting individual trial participants, is to advance 'generalisable knowledge' to help future patients. While that goal is laudatory, clinical research is fraught with ethical challenges including those that occur when research is conducted across multiple trial sites, in different countries or regions, in low-resource settings, in developing countries and with different, sometimes vulnerable, populations. The written clinical trial protocol is the appropriate instrument to illuminate, acknowledge and address ethical challenges specific to each individual study. However, writers of clinical trial protocols-members of the clinical research team in either industry, non-profit or academic settingsmay not have access to satisfactory single-source guidance to identify and address relevant ethical issues. The lack of guidance results in clinical trial protocols that either are silent on the ethical issues and choices made or include non-specific language about compliance with ethical principles without explicitly delineating such principles or challenges. In the absence of explicit description or discussion of ethical questions and choices, ethics committees (institutional review boards (IRBs) and research ethics committees (RECs), depending on the region) must identify the ethical issues implicit in the clinical trial protocol, infer how protocol writers addressed concerns and may assumewithout seeing evidence to the contrary-that ethical issues were not considered and appropriately managed. The lack of explicit description of, approach to and mitigation of ethical issues in a clinical trial protocol can result in time-consuming delay, as ethics committees pose questions that the writers must then answer in a later resubmission. Of even greater importance, not anticipating and planning for important ethical issues may potentially lead to problems in the trial itself.

To provide guidance and to raise the overall quality of clinical trial protocols, the Multi-Regional Clinical Trials Center of Brigham and Women's Hospital and Harvard (MRCT Center) ${ }^{\mathrm{i}}$ undertook the initial development of a Protocol Ethics Tool Kit ('Ethics Tool Kit'), accompanied by a guidance document with points to consider (available in fillable Microsoft Word format at http://mrctcenter.org/ resources/2014-11-14-training-material-mrct-ethicsessential-elements-and-points-to-consider-referencedocument-toolkit/). The intent of these resources is to help protocol writers recognise and address common ethical challenges in clinical trials, with specific attention to issues that arise in multinational settings. The Ethics Tool Kit is also intended to help ethics committees review and analyse clinical trial protocols in a more efficient, explicit and comprehensive manner.

\section{BACKGROUND}

Clinical trial protocols are central to the conduct of clinical trials and facilitate evaluation and review by key stakeholders, including regulators and ethics committees. ${ }^{1}$ Despite the importance of sound, wellwritten and ethical clinical trial protocols, existing

${ }^{\mathrm{i}}$ The MRCT Center was founded in 2009 to improve the design, conduct and oversight of multi-regional clinical trials, focusing on trials conducted in emerging economies and the developing world. The MRCT Center seeks to establish common, explicit, feasible and ethical standards for conduct of transnational clinical research (http://www. mrctcenter.org). 
guidelines for protocol writers have had limitations such as insufficient stakeholder involvement, lack of systematic development and weak empirical support. ${ }^{2}$ Two relatively recent documents provide a structure and define needed components of a clinical trial protocol, although neither focused specifically on the ethical issues raised by a planned study. The CONSORT (Consolidated Standards of Reporting Trials) Statement, updated in 2010, presents systematic evidence-based guidance for organising final study reports based on a checklist, and this can be used to inform protocol writing. ${ }^{3}$ CONSORT's checklist highlights 3 of the 11 elements contained in our Ethics Tool Kit, specifically the importance of proper study design, the choice of study population and the criticality of addressing potential harms. Similarly, the SPIRIT (Standard Protocol Items: Recommendations for Interventional Trials) 2013 Statement, generated by an international group of stakeholders, recommends minimum standards for inclusion in clinical trial protocols. ${ }^{1}$ SPIRIT includes two topics that might particularly require ethical consideration: the importance of informed consent and trial design, which are both included and broadened in our Ethics Tool Kit. Neither CONSORT nor SPIRIT comprehensively and directly addressed ethical issues in clinical trial protocols. The Ethics Tool Kit complements and expands these two prior documents by offering more focused guidance for identifying and treating ethical issues in clinical trial protocols.

\section{METHODS}

The development of the MRCT Center Ethics Tool Kit involved (1) formation of a working group, (2) literature review and (3) review of a sample of 100 approved clinical trial protocols. Following these initial steps, the multi-stakeholder working group aggregated, aligned and reviewed focused ethical questions that were then formatted as (4) an Ethics Tool Kit and accompanying guidance document to allow dynamic usage by protocol writers and ethics committees alike.

\section{FORMATION OF A WORKING GROUP}

A group of 20 experts from academic institutions (6), pharmaceutical companies (4), non-profit organisations (4), law firms (3) and ethics committees (3), with backgrounds in clinical trials, medicine, bioethics and law was formed by the MRCT Center in 2012 to create a list of ethical elements that should be addressed when writing and/or reviewing a clinical trial protocol. Each member introduced potential ethical elements by drawing upon the research ethics literature and existing sponsor protocol templates, areas identified as confusing by ethics committees and domestic and international guidelines. ${ }^{4-8}$ Initially, all recommendations from all 20 members were compiled. The ethics elements were then discussed, challenged and categorised to appropriately group similar elements and reduce redundancy. ${ }^{\text {ii }}$ The working group met a total of 24 times over a period of approximately 18 months by teleconference. One in-person meeting was also held to reach consensus on issues that could not be reconciled earlier. The 'Essential Elements' that comprise the basis of the Ethics Tool Kit were then compiled, annotated, reviewed and refined.

\section{LITERATURE REVIEW}

PubMed was searched to identify articles in English published from January 1995 to April 2015 that included

\footnotetext{
ii Nevertheless, not all elements are truly 'unique' and many are overlapping. For instance, a number of elements impact 'risk/benefit' even though risk/benefit is called out as a separate element.
}

recommendations for a list of essential ethical issues to consider when reviewing and/or drafting a clinical trial protocol. Search terms included: (1) ('clinical trial' [publication type] OR 'clinical trials as topic' [MeSH Terms] OR 'clinical trials' [All Fields]) AND ('ethics' [Subheading] OR 'ethics' [All Fields] OR 'ethics' [MeSH Terms]) NOT 'clinical trial' [Publication Type] and (2) clinical trials as topic $\{\mathrm{MeSH}$ Terms] AND protocol AND ethics NOT 'clinical trial' [Publication Type].

\section{CLINICAL TRIAL PROTOCOL REVIEW}

The working group undertook a descriptive review of a sample of 100 clinical trial protocols to determine if the Essential Elements drafted through consensus were present in current approved clinical trial protocols and, if they were, whether they were discussed directly from an ethical perspective. To minimise bias in the choice of clinical trial protocols to be evaluated and the review process, the review was conducted using a set of predefined guidelines. Protocols were selected based on the following criteria:

- Protocols that had been reviewed and approved by ethics committees

- Multi-site trials with at least one site outside of the USA

- Interventional trials, including medical, social/behavioural and devices

- Trials involving greater than minimal risk, as defined by US research regulations ${ }^{9}$

Selection was retrospective and consecutive from the start date, 30 June 2013, proceeding back in time until 100 clinical trial protocols matching the selection criteria were identified. Prior to selection, no protocol was reviewed for content (other than for the selection criteria listed above). Informed consent forms were also reviewed when available, as some of the Essential Elements might be addressed in the informed consent form instead of the protocol. Two authors reviewed each protocol; if there were disagreement on the assessment, a third author arbitrated. However, little disagreement between the two primary reviewers actually occurred; the kappa statistic, which measures inter-rater agreement, was 0.96 .

\section{RESULTS}

\section{Recommendations of the working group}

Although the assembled working group members had extensive involvement with various aspects of study design and protocol assessment, in their experience, clear and specific discussion of primary ethical issues in clinical trial protocols was unusual. This working group recommended that a dedicated ethics section be included in every protocol. Inclusion of such a section would (1) help clinical research teams proactively consider and articulate ethical considerations associated with their protocol and, as a result (2) improve the dialogue between ethics committees and clinical research teams and among clinical research team members themselves. No working group members were aware of a similar prior suggestion or exposition of how such a section should be structured.

Based on their collective expertise, and informed by the literature and protocol reviews, the MRCT Center work group identified 11 items (called Essential Elements; also see table 1) that should be considered for discussion in a dedicated ethics section within a clinical research protocol.

1. Addressing Relevant Question

2. Choice of Control and Standard of Care

3. Choice of Study Design

4. Choice of Subject Population

5. Potential Benefits and Harms 
Table 1 Essential Elements and survey results (sample size $=100$ protocols)

\begin{tabular}{ll}
\hline Essential Element & $\begin{array}{l}\text { Percent of protocols } \\
\text { covering element (\%) }\end{array}$ \\
\hline 1. Addressing relevant question & 96 \\
2. Choice of control and standard of care & 59 \\
3. Choice of study design & 44 \\
4. Choice of subject population & 39 \\
5. Potential benefits and harms & 76 \\
6. Informed consent & 56 \\
7. Community engagement & 9 \\
8. Return of research results and incidental findings & 49 \\
9. Post-trial access & 22 \\
10. Payment for participation & 40 \\
11. Study related injury & 43 \\
\hline
\end{tabular}

6. Informed Consent

7. Community Engagement

8. Return of Research Results and Incidental Findings

9. Post-Trial Access

10. Payment for Participation

11. Study Related Injury.

\section{Literature review}

Using the search criteria listed above, the literature review found only one relevant scholarly article that provided guidance for drafting and/or reviewing the ethical elements of a clinical trial protocol. The study was published in the psychiatry literature more than 15 years ago, and referenced the Research Protocol Ethics Assessment Tool (RePEAT), a 24-item checklist that contained some of the items identified in our work. ${ }^{4} 10$ Thus, we found little available guidance in a single organised format to guide which items should be considered for discussion in a protocol and how these considerations might be organised in a dedicated ethical section.

\section{Clinical trial protocol review}

A total of 100 clinical trial protocols were reviewed to determine if the 11 Essential Elements the working group drafted were present in the current approved clinical trial protocols and, if they were, whether they were discussed explicitly from an ethical perspective. A total of 40 clinical trial protocols were identified from publicly available published trials in the New England Journal of Medicine, 40 had been approved by independent central IRBs and were available to one of the working group members and 20 had been approved by academic IRBs and were available to one of the working group members. Of the 100 clinical trial protocols reviewed, 57 were funded by industry, 32 by governments, 3 by academic institutions, 3 by different combinations of the above categories and 5 were of indeterminate funding source.

As summarised in table 1, our list of 11 Essential Elements was variably addressed in these 100 clinical trial protocols. For example, while the first element, 'Addressing Relevant Question', was almost always included in a clinical trial protocol (96\%), other Essential Elements were mentioned much less frequently. It is not surprising that some Essential Elements such as Community Engagement or Post-Trial Access, for example, were mentioned in only $9 \%$ and $22 \%$ of protocols, respectively. Community Engagement and Post-Trial Access may not be relevant to some protocols, and the latter is, admittedly, an emerging issue. ${ }^{11}$ However, evidence of the thinking around Potential Benefits and Harms was not addressed in $24 \%$ of protocols, and Challenges in Informed Consent was not found in $44 \%$. Other Essential Elements that might be expected to be important for almost all protocols (Elements 2, $3,4,6,9,11$ ) were mentioned in $39 \%-59 \%$ of protocols. This variability may not be surprising as no regulation presently requires explicit discussion of ethical issues in written clinical trial protocols or informed consent forms. Absent regulatory requirements, study sponsors and funders may not dedicate resources to document the background thought processes in protocols.

The significance of these findings should not be overinterpreted. The lack of documentation of ethical considerations does not mean that the study was unethical, only that the thinking behind the choices made (eg, in study design, in study population choice, etc.) was not explicit. In addition, it does not imply that the ethics committee did not consider the ethical issues; the ethics committee meeting minutes were not reviewed nor were the exchanges, written or otherwise, between the ethics committee and the principal investigators. Further, the review itself of the 100 sampled protocols had limitations including the small sample size, the admittedly nonrepresentative nature of the protocols that were available and the use of non-validated review criteria. Some clinical trial protocols were analysed by representative(s) of organisations from which the protocols were obtained. This was necessary to protect confidentiality but may have introduced bias into the assessment process. In addition, no regulation presently requires explicit discussion of ethical issues in written clinical trial protocols or informed consent forms. Nonetheless, these findings suggest that critical ethical issues typically of serious concern to ethics committees are often not addressed explicitly in submitted clinical trial protocols.

\section{The Protocol Ethics Tool Kit: a tool to recognise and address ethical issues}

To ensure and reinforce adequate exposition of ethical issues within clinical trial protocols and to ease the burden of distilling and including this information, the MRCT Center working group developed the Protocol Ethics Tool Kit incorporating the Essential Elements. The Ethics Tool Kit was developed to (1) provide protocol writers and study teams with a tool to recognise and address common clinical trial ethical issues and (2) to

Table 2 Components of the MRCT Center's Protocol Ethics Tool Kit

\begin{tabular}{lll}
\hline Section & Description & For use by \\
\hline $\begin{array}{l}\text { Short Explanation of } \\
\text { the Ethics Essential } \\
\text { Elements }\end{array}$ & $\begin{array}{l}\text { A list of the 11 essential elements } \\
\text { to consider when writing or } \\
\text { reviewing a protocol with an } \\
\text { accompanying description of each } \\
\text { element }\end{array}$ & $\begin{array}{l}\text { Protocol writers, } \\
\text { ethics committees }\end{array}$ \\
Points to Consider & $\begin{array}{l}\text { Examples of detailed points to } \\
\text { consider for each of the Essential } \\
\text { Elements }\end{array}$ & $\begin{array}{l}\text { Protocol writers, } \\
\text { ethics committees }\end{array}$ \\
Examples of language from actual \\
Elinical trial protocols that \\
addressed a particular Essential & Protocol writers \\
Element & $\begin{array}{l}\text { Relevant citations and sources } \\
\text { References }\end{array}$ & Protocol writers \\
\hline
\end{tabular}


Table 3 Ethics Tool Kit in action-Essential Element 8: Return of Research Results and Management of Incidental Findings

\section{Points to consider}

- Address any planned disclosure of general (aggregated) research results, for example, posting of research results on ClinicalTrials.gov

- Address any planned disclosure of individual research results (IRRs) to subjects and the criteria or framework under which IRRs will be evaluated for returnability (or justify a 'no-return' approach, if applicable)

- Address any planned disclosure of incidental findings (IFs) to subjects and the criteria or framework under which IFs will be evaluated for returnability (or justify a 'no-return' approach, if applicable)

- If appropriate, include any proposed referral policies (ie, for confirmation of the IRRs or IFs and/or any necessary clinical care that might flow from the finding)

- Describe whether participants will have the ability to opt-in or opt-out of receiving IRRs and/or IFs, and any circumstances in which a participant's stated general preference to receive results will govern and/or a participant's preference not to be informed of IRRs and/or IFs will be overruled

\section{Scenarios}

- A spinal tumour detected through a research MRI where the protocol calls for the analysis of the image of an unrelated part of the body

- A genetic variant indicating a high risk of a certain type of cancer found during a whole-genome sequencing protocol where the focus of the research is limited to a different portion of the genome

- Genetic variants uncovered in the analysis of banked specimens and data under circumstances where the significance of the variant may have been unknown at the time the materials were banked, and the retrospective research was not targeting such variants ensure that ethics committees are able to evaluate clinical trial protocols comprehensively and efficiently. The Ethics Tool Kit is not intended to prescribe requirements, to limit ethical considerations or to impose mandates on how ethical issues must be addressed in a trial protocol. Rather, the Ethics Tool Kit is intended to guide thought and discussion and to ensure that ethical concerns specific to a clinical study are, at a minimum, considered in protocol development and made explicit in the protocol itself.

The Ethics Tool Kit is structured in such a way that it can be adapted to meet an individual user's needs and address specific challenges. Each Essential Element has (1) a short explanation, (2) specific points to consider, (3) background information, (4) practical examples and (5) references. Table 2 provides a brief description of the components of the Ethics Tool Kit and to whom they could be relevant. An online supplementary table S4 presents the short explanation and specific points to consider for each Essential Element. ${ }^{12-23}$ The Ethics Tool Kit in its entirety can be accessed at http://mrctcenter.org/resources/ 2014-11-14-training-material-mrct-ethics-essential-elements-andpoints-to-consider-reference-document-toolkit/.

Use of the Ethics Tool Kit may surface ethical issues that would be otherwise unexplored and also encourage rational, clearly articulated responses. For example, see table 3 on Essential Element 8: Return of Research Results and Incidental Findings.

The Ethics Tool Kit is not intended to serve as an exhaustive list of ethical issues that can occur in clinical research, and not every Essential Element is necessarily relevant to every protocol. However, it is recommended that protocol authors consider all Essential Elements, address those that are pertinent for the particular clinical trial and supplement as needed. Authors may choose to discuss ethics throughout the protocol, but the working group sees value in the practice of detailing ethics approaches in a dedicated 'Ethics Section' of the protocol.

\section{Computer-based training of the Ethics Tool Kit}

In February 2014, in an effort to disseminate the working group's efforts more widely to researchers in low-income and middle-income countries, the MRCT Center collaborated with colleagues at the Global Health Network at Oxford University, Oxford, England and adapted the Ethics Tool Kit for an innovative digital platform (https://globalhealthtrainingcentre. tghn.org/essential-elements-ethics/.) The Essential Elements were first reviewed by our collaborators and then tailored to the electronic format; meaningful visual components were added and assessments at the end of each module were integrated to gauge understanding. A total of 11 course modules corresponding to the 11 Essential Elements have been freely available online through the Global Health Training Centre since 2 December 2014. The uptake of the course has been higher than expected. As of 22 June 2015, the total number of modules taken was 3536 by 1024 users globally. The most commonly accessed modules were (1) Addressing the Relevant Question, (2) Choice of Control and Standard of Care, (4) Choice of Participant Population and (8) Return of Research Results/Incidental Findings. The e-learning course modules were reviewed for content and user-friendliness by the intended end users (investigators in low-income and middle-income countries) and by the leadership of the Global Health Network prior to public release. Furthermore, the Global Health Training Centre e-learning courses are formally recognised for quality and content ${ }^{\mathrm{iii}}$ by the Liverpool School of Tropical Medicine (http://www.lstmed.ac.uk/), the Bill and Melinda Gates Foundation (http://www.gatesfoundation.org/) and the Worldwide Antimalarial Resistance Network (http://www. wwarn.org/).

\section{DISCUSSION}

The Ethics Tool Kit has potential uses for individual protocol writers and study teams, study sponsors and ethics committees. For individual protocol writers and study teams, it provides a systematic and methodical approach to address the ethical implications of a planned clinical trial. This will assist protocol writers by alerting them to the important ethical issues in study design, enrolment and conduct of clinical trials, and will encourage articulation of appropriate ethical justification. The framework may be particularly valuable to those with less experience drafting clinical trial protocols. The guidance also may be used beyond the protocol, as it can prompt consideration of context-specific difficulties, pertinent policies and local regulatory requirements. For example, the Ethics Tool Kit may alert investigators in lowresource regions to consider challenges in assessing competencies of local sites, differing local medical standards and potential risks of exploitation of local and/or vulnerable populations.

For study sponsors and funders, the Ethics Tool Kit may be useful for documenting the nature of questions that were

iiihttps://globalhealthtrainingcentre.tghn.org/elearning/other-resources/

Li RH, et al. J Med Ethics 2016;42:229-234. doi:10.1136/medethics-2014-102540 
considered in protocol design and the analytical approach that formed the basis of the final design. Further, the Ethics Tool Kit may provide sponsors and study teams important insights into the research review process by delineating what research ethics committees are assessing when reviewing studies.

For ethics review committees, the review may be streamlined significantly by altering the protocol model to one in which the ethical reasoning is included in the original submission to the ethics committee. Without an explicit ethics discussion, an ethics committee is left to discern the ethical reasoning behind protocol decisions. When questions arise, the ethics committee engages the principal investigator in dialogue subsequent to the initial review and requests revisions or explanations that can result in significant delay to protocol approval. By altering the model to one in which the ethical reasoning is included in the original submission, dialogue between the sponsor/investigator and the ethics committee can be initiated upfront. The process would therefore become more efficient and ethics issues would be addressed proactively, directly and more completely.

There are limitations of the Ethics Tool Kit. There may be clinical trial questions that do not fit neatly into the framework we have developed, or the Ethics Tool Kit may be of limited utility when certain methodologies are used in clinical trials, particularly as those methodologies develop and change. For instance, adaptive clinical trials introduce the ethical dilemma of whether and when the investigator should disclose the results to date to prospective participants. If results are disclosed, later prospective participants may not wish to be randomised to what appears, with time, to be the inferior arm. Since this is an emerging issue and there is no international guidance on this dilemma, the working group did not address it in the Ethics Tool Kit.

The 11 Essential Elements are considered a starting point for protocol ethics discussion. Emerging concerns (eg, data transparency, publication policy, recruitment feasibility, innovative trial design) may result in future modifications. Feedback is being actively sought by the MRCT Center ${ }^{\text {iv }}$, so that the Ethics Tool Kit can continue to be refined and updated. Based on initial online use metrics, it appears that the Ethics Tool Kit is providing a needed educational resource for those seeking guidance on ethical protocol writing. An update is envisioned in 2017, and in early 2017, a survey will be deployed to uniformly collect user feedback on the value of the Ethics Tool Kit.

\section{CONCLUSION}

- Substantive discussion of specific ethical issues is rarely included in clinical trial protocols.

- A total of 11 'Essential Elements' have been identified that should be considered and addressed as appropriate in a clinical trial protocol.

- The Protocol Ethics Tool Kit has been developed to support protocol writers, study teams, sponsors, ethics committees and reviewers.

- Use of this tool could result in more efficient development and review of clinical trial protocols and may result in wider appreciation of the ethical challenges in clinical research.

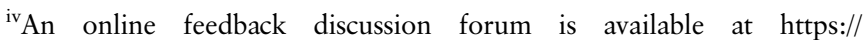
bioethicsresearchreview.tghn.org/community/groups/group/essential_ elements/ that captures comments in real time.
}

\section{Author affiliations}

${ }^{1}$ Multi-Regional Clinical Trials Center of Brigham and Women's Hospital and Harvard, Boston, Massachusetts, USA

${ }^{2}$ Janssen R\&D, Johnson \& Johnson, Raritan, New Jersey, USA

${ }^{3}$ Ropes \& Gray LLP, Boston, Massachusetts, USA

${ }^{4}$ The Global Health Network/Oxford University, Oxford, UK

${ }^{5}$ Quality Assurance, AbbVie, Chicago, Illinois, USA

${ }^{6}$ WCG (WIRB- Copernicus Group), Olympia, Washington, USA

${ }^{7}$ Health Care Group, Verrill Dana LLP, Boston, Massachusetts, USA

${ }^{8}$ Public Responsibility in Medicine and Research, Boston, Massachusetts, USA

${ }^{9}$ Petrie-Flom Center, Harvard Law School, Cambridge, Massachusetts, USA

${ }^{10}$ Morgan Lewis, New York, NY, USA

${ }^{11}$ NYU Langone Medical Center, New York, New York, USA

${ }^{12}$ Colorado School of Public Health Anschutz Medical Campus Aurora, CO

${ }^{13}$ Eli Lilly and Company, Indianapolis, Indiana, USA

${ }^{14}$ International AIDS Vaccine Initiative, New York, New York, USA

${ }^{15}$ Harvard School of Public Health, Boston, Massachusetts, USA

${ }^{16}$ Sanofi, New York, New York, USA

${ }^{17}$ Multi-Regional Clinical Trials Center, Brigham and Women's Hospital and Harvard Medical School, Boston, Massachusetts, USA

Contributors The paper originated from the work of a multi-stakeholder group convened by the Multi-Regional Clinical Trials Center of Brigham and Women's Hospital and Harvard (MRCT Center) to develop ethical principles for writers and reviewers of protocols for clinical trials that involve emerging economies. Participants in the working group were self-selected based on relevant expertise and were selffunded. The MRCT Center is supported by voluntary contributions from a variety of entities as well as grants (see http://mrctcenter.org/about-mrct/funding-andsupport/). Each of the authors participated in convened meetings that formed the basis of the content, and contributed to writing one or more sections of the toolkit described in the article; each author reviewed a draft of and the final submitted manuscript. The manuscript itself was written and revised by the first two and last two authors listed. The guarantor is Barbara E. Bierer, MD Professor of Medicine, Harvard Medical School and Faculty Co-chair of the MRCT Center; she retains final responsibility for the content.

Competing interests None declared.

Provenance and peer review Not commissioned; externally peer reviewed.

Open Access This is an Open Access article distributed in accordance with the Creative Commons Attribution Non Commercial (CC BY-NC 4.0) license, which permits others to distribute, remix, adapt, build upon this work non-commercially, and license their derivative works on different terms, provided the original work is properly cited and the use is non-commercial. See: http://creativecommons.org/ licenses/by-nc/4.0/

\section{REFERENCES}

1 Chan A-W, Tetzlaff JM, Gotzsche PC, et al. SPIRIT 2013 explanation and elaboration: guidance for protocols of clinical trials. BMJ 2013;346:e7586.

2 Tetzlaff JM, Chan A-W, Kitchen J, et al. Guidelines for randomized controlled trial protocol content: a systematic review. Syst Rev 2012;1:43.

3 CONSORT. The CONSORT statement. 2010. http://www.consort-statement.org/ consort-statement/overview0/

4 Roberts LW, Geppert CMA, Brody JL. A framework for considering the ethical aspects of psychiatric research protocols. Compr Psychiatry 2001;42:351-63.

5 Emanuel EJ, Wendler D, Grady C. What makes clinical research ethical? JAMA 2000;283:2701-11.

6 World Medical Association. Declaration of Helsinki: ethical principles for medical research involving human subjects. JAMA 2013;310:2191-4.

7 National Commission for the Protection of Human Subjects of Biomedical and Behavioral Research. The belmont report. Fed Regist 1979;44:23192-7.

8 Council for International Organizations of Medical Sciences. International ethical guidelines for biomedical research involving human subjects, revised draft. Geneva: CIOMS, 2002. http://www.cioms.ch/frame_guidelines_nov_2002.htm (accessed 2 Apr 2014)

9 Protection of Human Subjects, 45 C.F.R. § 46.102. 2009.

10 Roberts LW. Ethical dimensions of psychiatric research: a constructive, criterion-based approach to protocol preparation. The Research Protocol Ethics Assessment Tool (RePEAT). Biol Psychiatry 1999;46:1106-19.

11 Grady C. The Challenge of assuring continued post-trial access to beneficial treatment. Yale J Health Policy Law Ethics 2005;5:425-35.

12 London AJ. The ambiguity and the exigency: clarifying "standard of care" arguments in international research. J Med Philos 2000;25:379-97.

13 Van der Graaf R, Roes KCB, van Delden JJM. Adaptive trials in clinical research: scientific and ethical issues to consider. JAMA 2012;307:2379-80. 


\section{Clinical ethics}

14 Davis AL. The study population: women, minorities and children. In: Amdur RJ, Bankert EA, eds. institutional review board: management and function. $2 \mathrm{~d}$ edn. Sudbury, MA: Jones and Bartlett Publishers, 2006:129-33.

15 Emanuel EJ, Wendler D, Grady C. An ethical framework for biomedical research. In: Emanuel EJ, Grady C, eds. The Oxford textbook of clinical research ethics. New York: Oxford University Press, 2008:123-35.

16 International Conference on Harmonisation of Technical Requirements for Registration of Pharmaceuticals for Human Use (ICH). Guidance for industry E6 good clinical practice: Consolidated guidance. Department of Health and Human Services. 1996. See §4.8.

17 Weijer C, Goldsand G, Emanuel EJ. Protecting communities in research: current guidelines and limits of extrapolation. In: Emanuel $E$, Crouch $R$, Grady C, et al. Ethical and regulatory aspects of clinical research: readings and commentary. Baltimore: The Johns Hopkins University Press, 2003:340-1.
18 Zawati MH, Knoppers BM. International normative perspectives on the return of individual research results and incidental findings in genomic biobanks. Genet Med 2012;14(4):484-8.

19 Miller F, Mello M, Joffe S. Incidental findings in Human Subjects Research: what do investigators owe research participants? Journal of Law, Medicine, and Ethics: 36.2271-79.

20 Sofaer N, Strech D. Reasons why post-trial access to trial drugs should, or need not be ensured to research participants: a systematic review. Public Health Ethics 2011:4:160-84.

21 Association of the British Pharmaceutical Industry (ABPI). Clinical trial compensation guidelines. ABPI. 1994.

22 Beh H. Compensation for research injuries. IRB 2005;27:11-15.

23 Guest $\mathrm{S}$. Compensation for subjects of medical research: the moral rights of patients and the power of research ethics committees." J Med Ethics 1997;23:181-5 


\section{Correction}

Li R, Wacholtz MC, Barnes M et al. Incorporating Ethical Principles into Clinical Research Protocols: A Tool for Protocol Writers and Ethics Committees. J Med Ethics doi:10.1136/medethics-2014-102540

The author affiliations for this article have been corrected to:

Rebecca H Li, ${ }^{1}$ Multi-Regional Clinical Trials Center of Brigham and Women's Hospital and Harvard, Boston, Massachusetts, USA

Mary C Wacholtz, ${ }^{2}$ Janssen R\&D, Johnson \& Johnson, Raritan, New Jersey, USA

Mark Barnes, ${ }^{1,3}$ Multi-Regional Clinical Trials Center of Brigham and Women's Hospital and Harvard, Boston, Massachusetts, USA; Ropes \& Gray LLP, Boston, Massachusetts, USA

Liam Boggs, ${ }^{4}$ The Global Health Network/Oxford University, Oxford, UK

Susan Callery-D’Amico, ${ }^{5}$ AbbVie, North Chicago, Illinois, USA

Amy Davis, ${ }^{6}$ formerly with Public Responsibility in Medicine and Research, Boston, Massachusetts, USA

Alla Digilova, ${ }^{10}$ Morgan, Lewis \& Bockius LLP, New York, NY, USA

David Forster, ${ }^{7}$ WCG (WIRB- Copernicus Group), Puyallup, Washington, USA

Kate Heffernan, ${ }^{8}$ Verrill Dana LLP, Boston, Massachusetts, USA

Maeve Luthin, ${ }^{8}$ Public Responsibility in Medicine and Research, Boston, Massachusetts, USA

Holly Fernandez Lynch, ${ }^{9}$ Petrie-Flom Center, Harvard Law School, Cambridge, Massachusetts, USA

Lindsay McNair, ${ }^{6}$ WCG (WIRB- Copernicus Group), Princeton, New Jersey, USA

Jennifer E Miller, ${ }^{11}$ NYU School of Medicine; Bioethics International, New York, USA

Jacquelyn Murphy, ${ }^{12}$ Colorado School of Public Health Anschutz Medical Campus Aurora, CO

Luann Van Campen, ${ }^{13}$ Eli Lilly and Company, Indianapolis, Indiana, USA

Marc Wilenzick, ${ }^{14}$ International AIDS Vaccine Initiative, New York, New York, USA

Delia Wolf, ${ }^{15}$ Harvard School of Public Health, Boston, Massachusetts, USA

Cris Woolston, ${ }^{16}$ Sanofi, New York, New York, USA

Carmen Aldinger, ${ }^{1}$ Multi-Regional Clinical Trials Center of Brigham and Women's Hospital and Harvard, Boston, Massachusetts, USA

Barbara E Bierer, ${ }^{17}$ Multi-Regional Clinical Trials Center, Brigham and Women's Hospital and Harvard Medical School, Boston, Massachusetts, USA

\section{(6) \\ OPEN ACCESS}

Open Access This is an Open Access article distributed in accordance with the Creative Commons Attribution Non Commercial (CC BY-NC 4.0) license, which permits others to distribute, remix, adapt, build upon this work non-commercially, and license their derivative works on different terms, provided the original work is properly cited and the use is non-commercial. See: http://creativecommons.org/licenses/by-nc/4.0/

J Med Ethics 2016;42:449. doi:10.1136/medethics-2014-102540corr1 